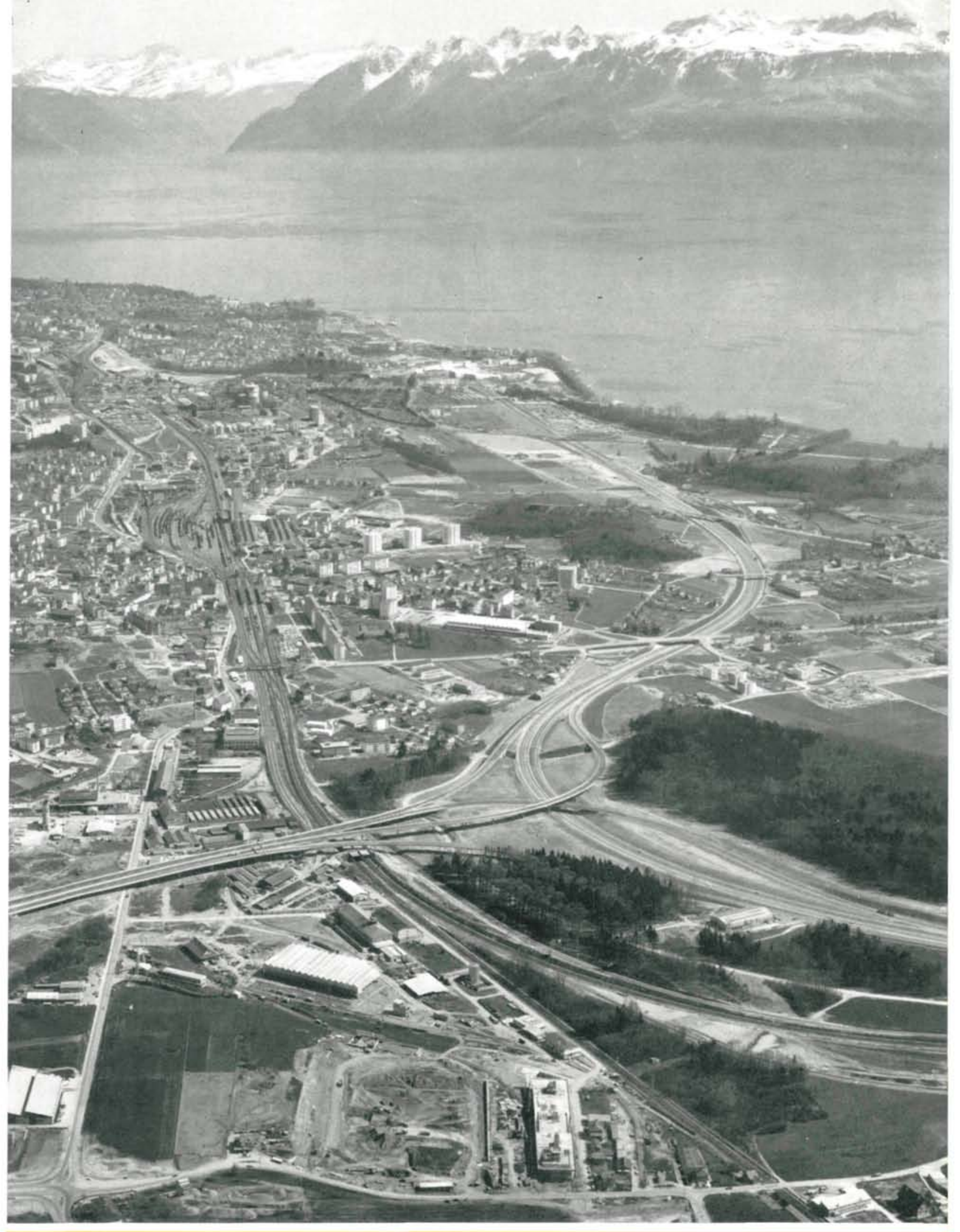

\title{
intercambio direccional en roseta - Suiza
}




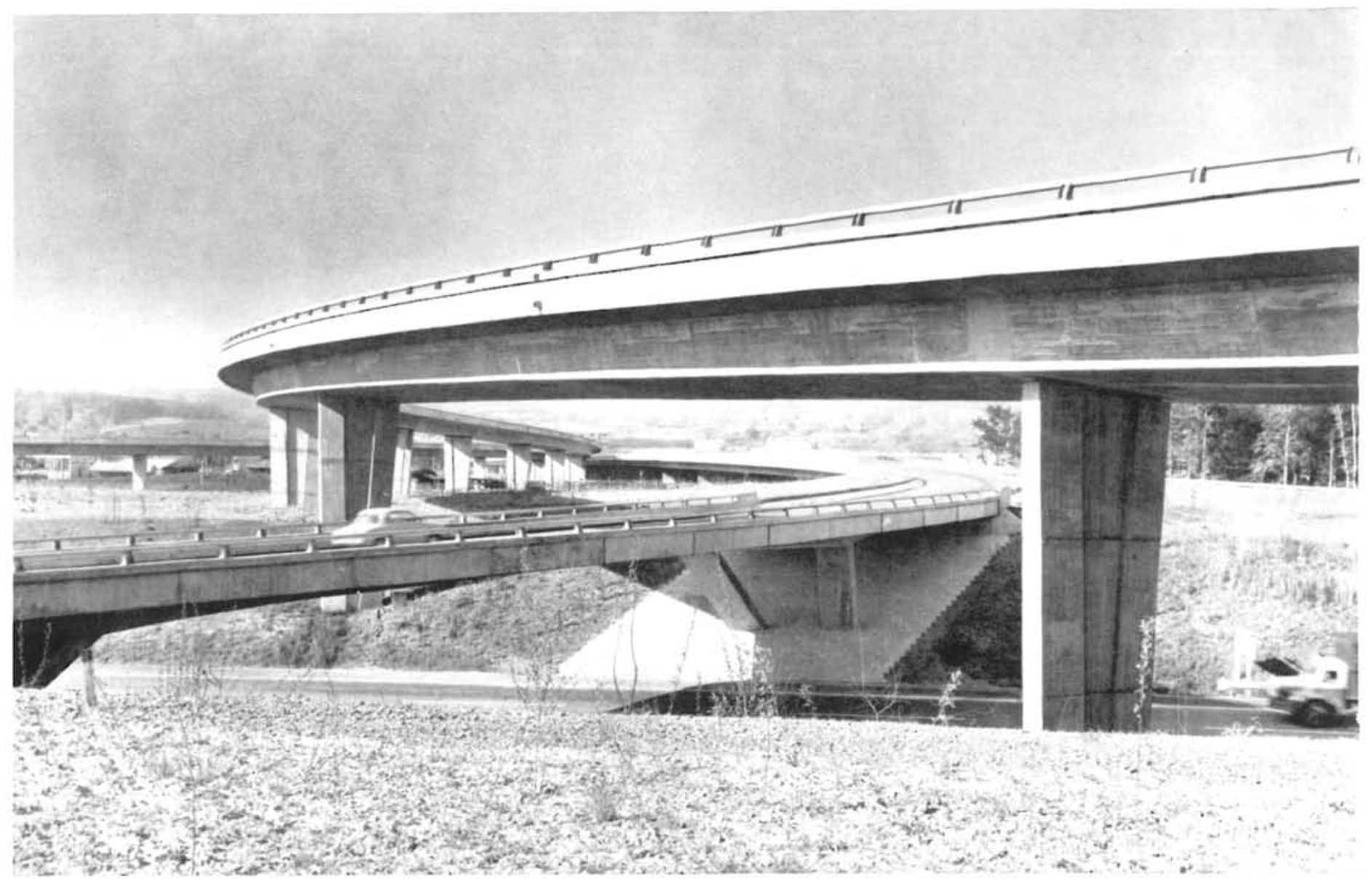

Paso intermedio.

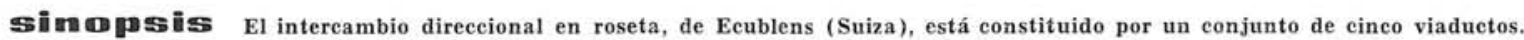

La multiplicidad de vías que debía salvar la autopista Lausana-Berna planteó un problema importante, ya que era preciso tener en cuenta factores de tipo técnico, en lo que respecta a las obras de fábrica; estéti-

Técnicamente, $y$ teniendo en cuenta que las condiciones del terreno eran poco apropiadas para levantar obras pesadas, la solución adoptada consiste, en esen cia, en cínco viaductos que constituyen otros tantos pasos, algunos de ellos intermedios, es decir, situados entre dos vías: una superior y otra inferior.

Cada uno de los pasos se ha subdividido en varios tramos de viga cajón continua. Las dos pilas centrales de cada tramo son rigidas, para poder absorber los efectos del viento; las demás están articuladas en la parte superior $\mathbf{y}$ en la inferior, $\mathbf{y}$ todas ellas tienen forma trapezoidal invertida. Entre trozos continuos se han previsto juntas de dilatación, con un dispositivo metálico que asegura la continuidad de la calzada $y$ con una capacidad de libre movimiento longitudinal de unos 16 centímetros.

Debido a las pobres condiciones resistentes del terre no fue preciso que cierto número de soportes se reforasen con la hinca de pilotes, ganando asi mayor capacidad de sustentación.

Uno de los pasos discrepa de la tónica general, ya que consiste en un tramo con bielas como soportes interme. dios. La estética fue la finalidad primordial perseguida con esta variante. 


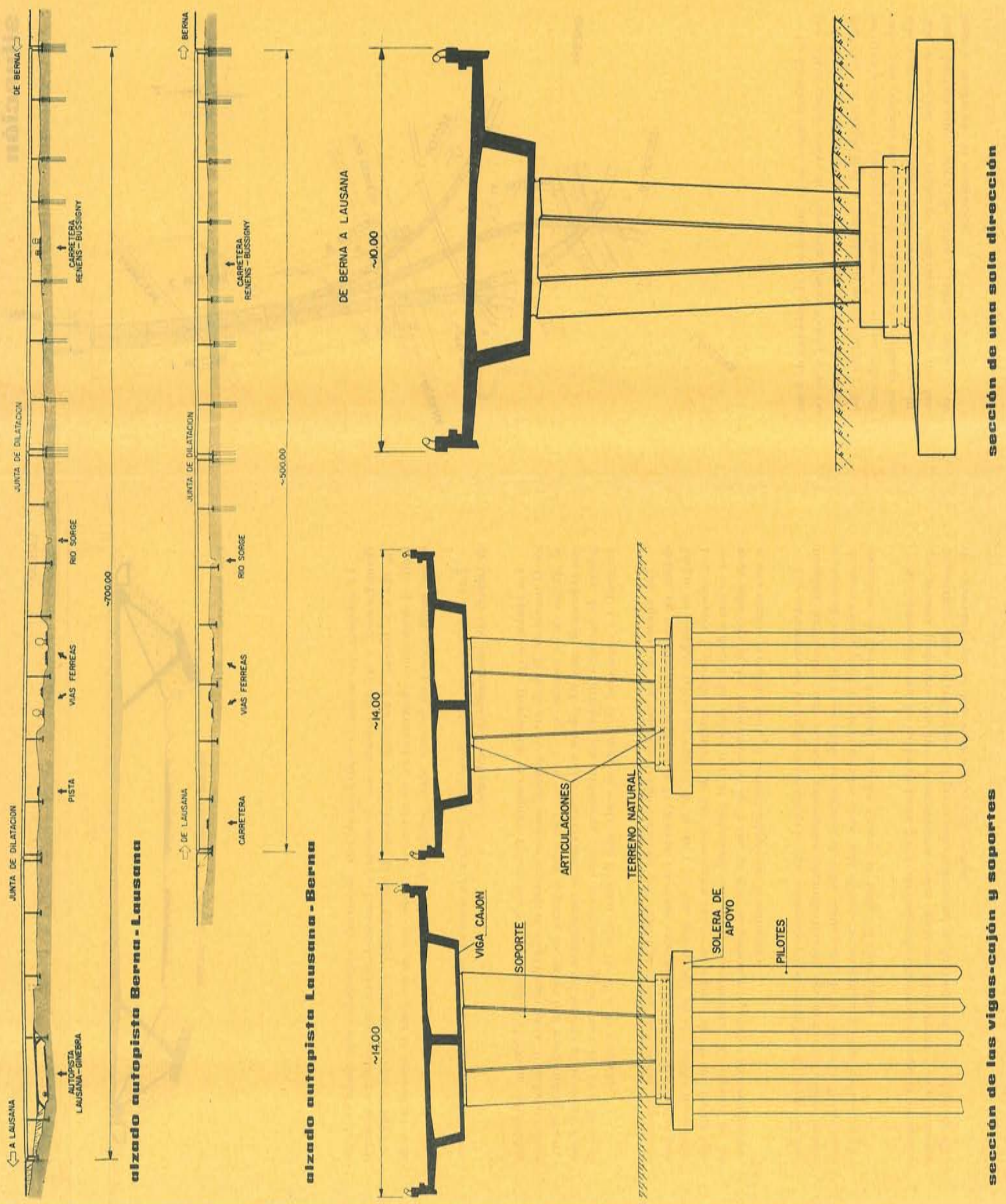



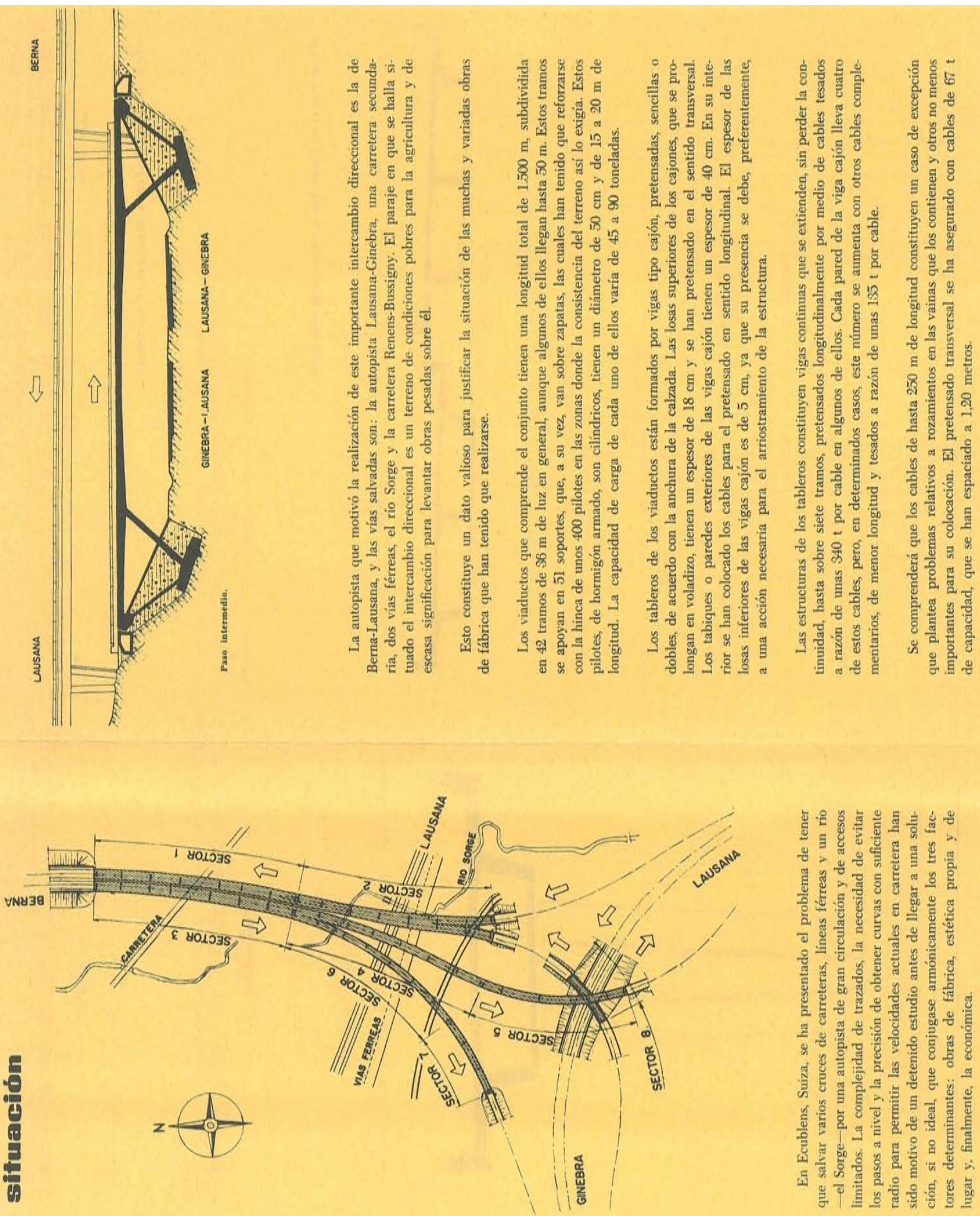


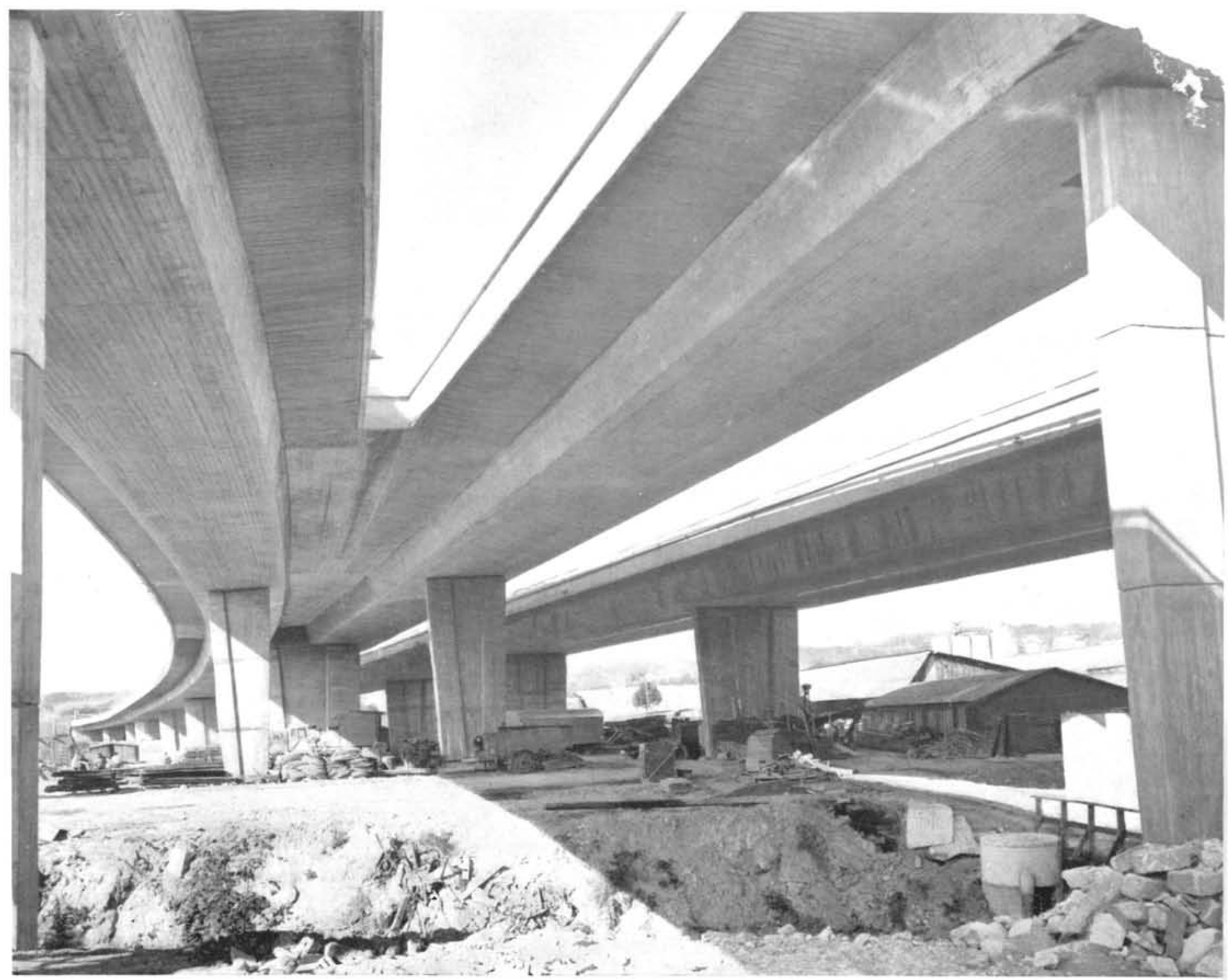

Bifurcación de direcciones.

Los soportes de los distintos tramos tienen forma de pared plana, trapezoidal, de base más estrecha que la parte superior. Con objeto de permitir el acortamiento de las vigas que la retracción motiva en conjunción con los efectos del pretensado y variación de temperatura, los soportes se han articulado en su base y en la parte superior. Estas articulaciones se han protegido con hormigón zunchado de un espesor de $10 \mathrm{~cm}$. En cada uno de los trozos continuos se ha dejado un tramo con soportes rígidos capaz de absorber, por sí solo, los efectos de frenado. Los dos soportes de estos tramos se han empotrado en los cimientos.

Con objeto de que los soportes se hallen aplomados, a pesar de los acortamientos experimentados por el tablero debido a las causas anteriormente apuntadas, ha sido necesario tomar la precaución de inclinar dichos soportes proporcionalmente a su distancia al tramo central, rígido, antes de proceder al hormigonado de la estructura del tablero. 
La continuidad de la superficie de rodadura entre dos trozos continuos se ha asegurado por medio de juntas de dispositivo metálico, capaces de absorber variaciones de longitud de unos 16 centímetros.

Los antepechos tienen una forma estudiada que se asemeja a una guía deslizante de seguridad, que se ha hormigonado después de haber tesado las armaduras de pretensado de los viaductos. La parte superior de estos antepechos se compone de un pasamanos tubular, de aleacción metálica ligera, y tiene $13 \mathrm{~cm}$ de diámetro.

La parte hueca interior de las vigas cajón es visitable, y en ella se han colocado las canalizaciones de agua, eléctricas, de alumbrado, telefónicas y cables de alta tensión. Las bajantes para el desagüe de aguas pluviales se han disimulado en el interior del cuerpo de los soportes.

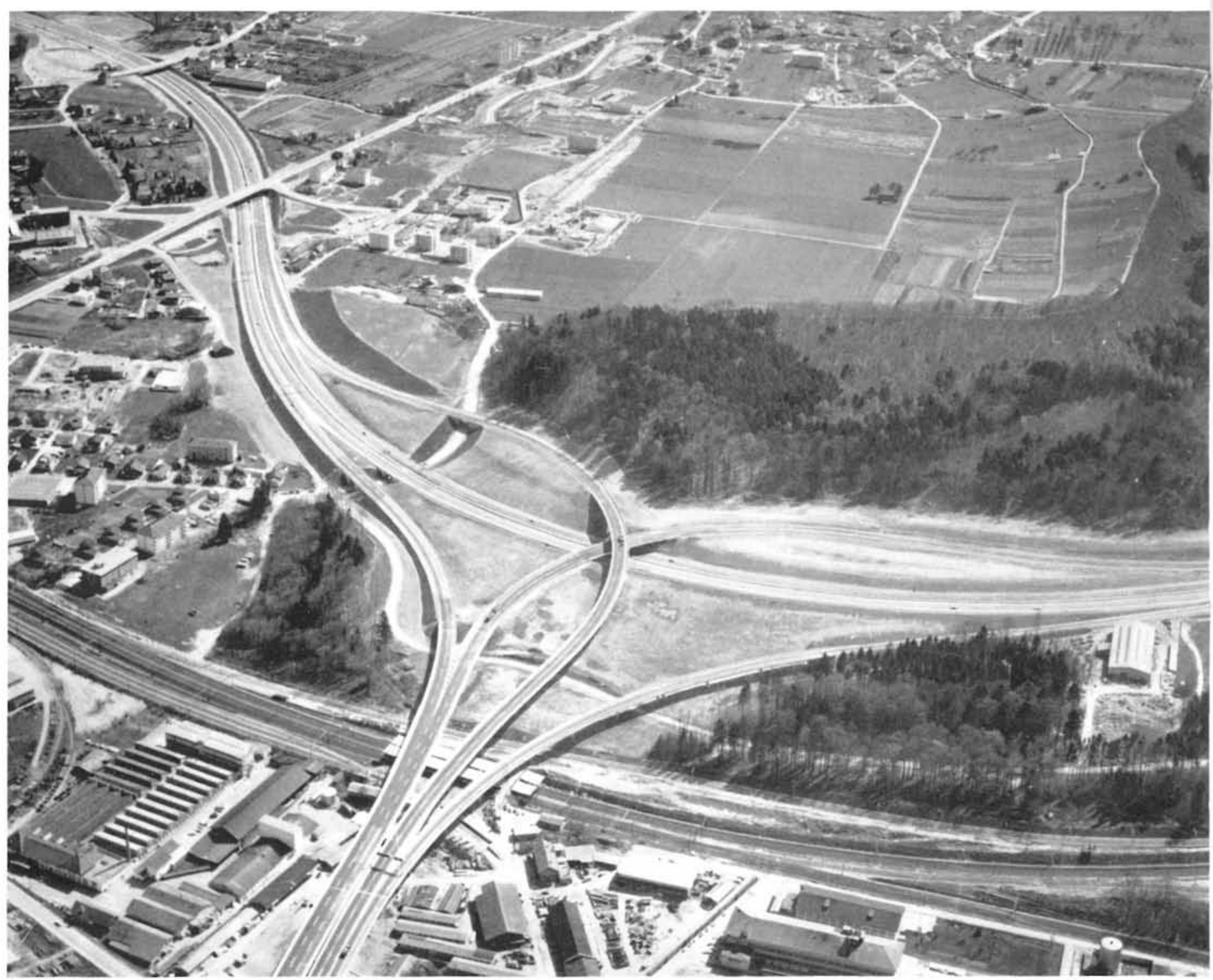




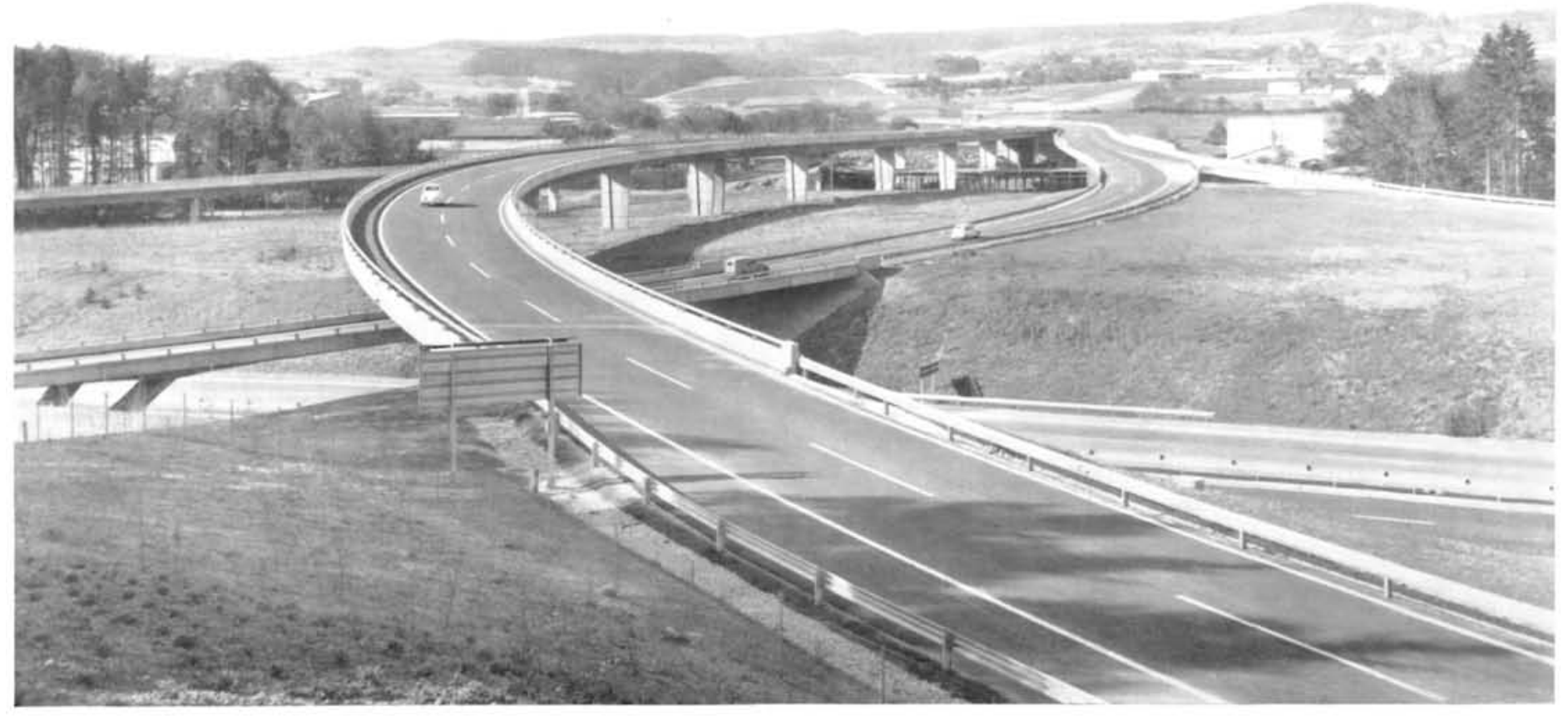

El paso intermedio para una pista superior sobre la autopista de Ginebra a Berna e inferior a la de Berna a Lausana, ha sido concebido de forma distinta a los viaductos anteriormente descritos por razones puramente estéticas. Este paso tiene su eje en esviaje, en curva, se ha peraltado y su estructura se ha sostenido por medio de bielas. El tablero está constituido por una losa maciza de hormigón pretensado. La disposición de los elementos que componen su estructura le dan la gracia y el efecto de una obra notablemente aligerada.

El estudio analítico de las distintas estructuras ha creado problemas complejos de cálculo que se han resuelto con ayuda de una calculadora electrónica.

Como los distintos trazados exigían curvas distintas en planta y elevación, y peraltes y anchuras diferentes de calzada, particularmente en las bifurcaciones de las bandas de circulación, la ejecución de las obras también ha presentado sus problemas y complicaciones, pues fue necesario mantener el tráfico de todas las vías cruzadas durante el período de construcción, y en particular el de las líneas férreas, ya que se disponía de poca altura para hacer frente a las medidas de seguridad que todo paso superior a una vía férrea requiere.

El estudio y dirección de obras se han confiado a la asociación de dos Estudios: uno, el de Bonnard \& Gardel, de Lausana, y Soutter \& Schalcher, de Zurich. 


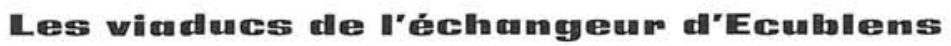

François Guisan, ingénieur.

L'échangeur routier construit à Ecublens, Suisse, est formé par cinq viaducs.

La multiplieité de voies qui devaient franchir l'autoroute Lausanne-Berne posa un problème importønt; il fallait tenir compte des facteurs de type technique, en ce qui concerne les travaux de maçonnerie, esthétiques, quant à l'harmonie avec le paysage local, et économiques.

Techniquement et compte tenu des conditions du terrain peu appreprié à l'exécution d'ouvrages lourds, la solution 2doptée consiste, essentiellement, en cinq viadues qui constituent autant de voies de circulation-quelques-unes intermédiaires, c'est-à-dire, situées entre deux voies: une supérieure et une inférieure.

Chaque passage se divise, à son tour, en plusieurs travées de poutre caisson continue. Les deux piles centrales de chaque travée sont rigides, pour pouvoir absorber les effets du vent; les autres piles sont articulées à leur partie supérieu rion munis d’un dispositif métallique qui assure la continuité de la chaussée et avec une capacité de libre mouvement longitudinal d'environ 16 centimètres.

A cause des faibles conditions de résistance du terrain, il a été nécessaire qu'un certain nombre de palées soit renforcé à l'aide de pieux, assurant ainsi une plus grande capacité de sustentation.

Seul un des ponts diffère de Ia tenue générale des ouvrages. C'est un pont intermédiaire, biais, qui s'appuie sur des béquilles. L'esthétique à été le motif principal de cette variante.

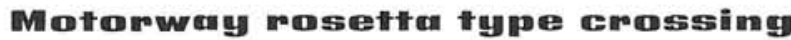

François Guisan, engineer.

This crossing, at Ecublens, Switzerland, involves five viaducts. The number of roads that the Lausanne-Berne motorway had to cross presented a difficult problem, since technical, aesthetic ane economic quastions had to be considered, all on a complex scale.

As the soil was not suitable to support heavy struetures, it was decided to build five viaducts, each providing a traffic route. Some of these viaducts cross each other at three levels.

The viaducts consist of box girder sections, joined together to form continuous beams. The two central piles of ezch section are rigid, to withstand wind forces, and the others are hinged at the top and bottom. The sections are separated by dilatation joints, fitted with isplacement of $16 \mathrm{~cm}$.

Some of the supports were placed on pile foundations, to overcome the weak bearing strength of the subsoil.

One of the road sections did not have any rigid central supports, and this arrangement was chosen for aesthetic reasons.

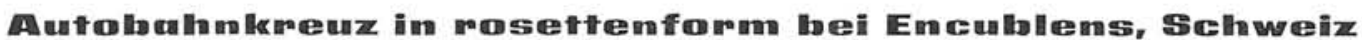

François Guisan, Ingenieur.

Das Autobahnkreuz bei Ecublens, Schweiz, besteht zus fünf Brücken.

Die grosse Anzahl von Strassen, die die Autobahn von Lausanne nach Bern zu überqueren hatte, brachte einige Schwie rigkeiten mit sich, denn man musste rein technische Faktoren, wie die Errichtung von Fabriken, ästhetische, wie Harmonie mit der Landschaft und schliesslich auch finanzielle Gesichtspunkte in Betracht ziehen.

Mit Rücksicht auf die Bodenbedingungen, auf Grund derer man dort keine schweren Bauwerke errichten durfte, kam man vom technischen Standpunkt aus gesehen, zu folgender Lösung:

Man sah 5 Autobrücken vor, die zum Teil selbst Ubergänge darstellen oder zwischen zwei anderen Brücken verlaufen, die darüber und darunter liegen.

Jede Brücke ist in mehrere Abschnitte eingeteilt und besteht aus einem durchgehenden Kasettenträger. Die zwei Mittelpfeiler jedes Abschnittes sind steif, um die Wirkungen des Windes aufzufangen; die übrigen besitzen Gelenke im oberen und unteren Teil. Alle haben die Form eines umgekehrten Trapezes. Bei lang durchgehenden Abschnitten hat man Dehnungsfugen mit einer Metallvorrichtung von $16 \mathrm{~cm}$ Längsspielraum vorgesehen, die Unterbrechungen in der Fahrbahn

Auf Grund der schlechten Bodenbedingungen war die Verstärkung einer gewissen Anzahl von Pfeilern durch das Einrammen von Pfählen notwendig, um eine Steigerung des Tragvermögens zu erreichen.

Eine der Brücken unterscheidet sich von den übrigen in einem Abschnitt, der durch Zugstangen an Stelle von Pfeilern getragen wird. Mit dieser Änderung hat man hauptsächlich der Ästhetik Rechnung tragen wollen. 